\title{
Virtual Reality Technology in Visual Design of Artistic Images: Analysis and Applications
}

\author{
Ling He $\mathbb{B}$ and Shanshan Zhu \\ School of Art \& Design, Shaanxi University of Science \& Technology, Xi'an, Shaanxi 710000, China \\ Correspondence should be addressed to Shanshan Zhu; zhuss2021@126.com
}

Received 27 October 2021; Revised 21 December 2021; Accepted 23 December 2021; Published 11 January 2022

Academic Editor: Rahman Ali

Copyright (C) 2022 Ling He and Shanshan Zhu. This is an open access article distributed under the Creative Commons Attribution License, which permits unrestricted use, distribution, and reproduction in any medium, provided the original work is properly cited.

\begin{abstract}
Within a few decades of development, Internet is been refreshing the world's awareness of its development and potentials at an unexpected speed. In recent years, virtual reality technology has begun to be applied to more and more fields. Especially in instant transmission of information, the applications of VR are incomparable. Akin to the wide-ranged advantages of the technology in education and health, the applications of VR in the art of artistic image designing need to be addressed. With this research work, a comprehensive study is presented about the applications of virtual reality in the art of image visualization. The research intends to analyze the possibility of intermingling modern image visualization with the emerging VR technology. Following a systematic approach, the history and development of display design are reviewed besides studying the artistry techniques used in the technology of art image visualization design. The interdisciplinary association of the two fields is explored with the help of relevant algorithms. The model proposed in the paper for the design of art image is based on the visual interactive process, rigorous assembly sequence, and appropriate equipment mode. As a result, the whole design process is more controllable and feasible. The suggested design concept is verified dynamically. Through the collection and comparison of feedback information, the hypothesis algorithm of the model is updated to accommodate the dynamic information so that to reflect the complete image design process. To assess performance of the model, evaluation was conducted from various perspectives. The three basic image operations such as illumination change, occlusion change, and color change were performed by using the two technologies such as the traditional image design and VR-based image design. From the comparison of experimental data, it was revealed that the effects of traditional art image visualization products produced by the art image visualization and that of VR technology are quite different. For instance, the visualization score achieved for the lighting change operation using the VR technology was $95.48 \%$, whereas $85.57 \%$ was obtained for the same operation using the traditional image design technology. Similarly, while adding visual effects using the VR technology, an average of $9 \%$ higher score was obtained for the said image operations. The promising results attained for the VR-based design testify that the technology is feasible and suitable to be intermingled with the visual design of art image. As a whole, the findings justify that the proposed approach is quite applicable in visual design of art image systems. In view of the significance of the research, the study can be extended to related domains of computer-based designing and visualization as well.
\end{abstract}

\section{Introduction}

In today's society, information technology is changing the traditional way of life and the habits of people [1]. First time in the history of human beings, it has become possible to browse the events, from anywhere, that have happened or going to happen $[2,3]$. Nowadays, application of VR technology is getting more attention owing to its unprecedented support for authenticity, interactivity, and comprehensiveness. Like in other domains, the technology offers unlimited development prospects in the field of art image visualization design $[4,5]$. People of the present era experience more fun and entertainment through this synthetic, integrated, networked, and intelligent art-plus-technology [6,7].

Keeping in view the wide-spread application value of $\mathrm{VR}$, research interest in using the technology of VR in the 
field of art image visualization design is on the rise [8]. Especially in the European and American countries where information technology is more developed, many leading designers have tried to extensively use this technology. Aim behind using the technology in art designing is to mimic the functions of related groups in the toolkit. This is to make possible innovative construction in the process of architectural design. Additionally, a more realistic view of simulation and animation is ensured. For example, at the end of 2017, Adobe launched a Sensei deep learning system. The system can be widely compatible with the contemporary image design systems and support pixels-based analysis of various elements and materials. The system can comprehensively analyze all things in the photo and process and select the material according to the needs of customers [9]. Although the domestic research on the application of this technology is relatively late, it has also made remarkable achievements. Recently, many organizations have begun to utilize this kind of technical means to show their design ideas and to achieve more vivid effects than the traditional mode [10]. The research teams of universities such as Beihang, Tsinghua, Zhejiang University, and Xi'an Aerospace have made great achievements in their respective fields. In addition, Lin Dong introduced the technology of VR into the campus design, which greatly improved the overall planning of the campus landscape [11]. Dong Han used VR technology in the designing of school related activities, which effectively promoted the cooperation between related disciplines [12].

This paper studies the application of computer virtual reality technology in art image visualization design. The characteristics of artistic display are studied in general and the technical characteristics of modern art image visualization design in particular to assess the use of VR technology in the realm of art image visualization design. The paper precedes to compare the analysis with the traditional art image visualization design in the virtual environment. The research is of high significance in the areas of art image design and image visualization. Moreover, the study sets a platform to combine the two technologies such as art image visualization design and VR.

\section{Related Work}

In this section, a detail discussion is hereby presented about VR, Artificial Intelligence (AI), and visual design of art image.

2.1. Virtual Reality and Artificial Intelligence. VR refers to a three-dimensional synthetic environment that simulates a real or imaginary scenario in such an engrossing way that the digital world is perceived as a real one. It integrates a variety of modern information technologies, enhances simulation effect, and works on the user's senses. The main categories of VR systems are nonimmersive, immersive, augmented reality, and distributed virtual reality. The main difference between these categories lies in the different equipment used and/or the working methods followed. The key factor in all these categories is virtuality while the degree of virtuality distinguishes a particular VR system from the rest. Among the said categories, the distributed VR supports the real-time cooperation of multiusers across the digital space. This opens up a new band of the technology that can be easily extended to other domains and, therefore, has a higher dimension of applications [13].

Artificial intelligence (AI) originated from computer application has been among the top technologies since the last century. Although the technology has been extensively used for more than 40 years and by dint of which the rapid development of computer technology became possible, it still appears to have infinite potential. Scientifically speaking, AI is about making computer such that to play the role of human being, that is, using information terminals to simulate human thinking process. The technology involves wide-range categories, covering not only the emerging computer science but also the traditional knowledge system of human beings. Therefore, it is pertinent to say that "it links the natural science and social science." Especially in the design of advertising posters sponsored by capital, there is a huge burst of energy. With a span of a few days, the technology can be used to design hundreds of millions dedicated and distinctive posters.

\subsection{Significance of VR in the Field of Artistic Image Visual} Design. The rapid development of VR technology has attracted the attention of many designers. Some powerful companies and well-known teams have joined the ranks and trying to use the technology to innovate the way of product display. The role of VR technology in the field of artistic visual design can be summarized in the following points. (1) With the VR technology, the designer's working environment can be optimized in such a way that the work efficiency is drastically improved. Moreover, the designer's design idea can be directly displayed through a vivid and easy-to-understand three-dimensional model. The model can be reused and/or updated without making the actual model from the scratch. (2) The technology can effortlessly improve the virtual assembly function needed in the field of visual design. With the help of VR, a designer can get the data about the work at the right time or as and when needed. Besides, through postprocessing, data can be refined to avoid possibilities of malfunctions. However, as these functions involve many other disciplines, a variety of technologies are needed to be incorporated accordingly.

2.3. Virtual Reality in Art Design Visualization. VR is the use of computer-based simulation enabling a user to interact with a three-dimensional synthetic world. Visualization refers to technique of designing drawings, images, and/or animations to communicate a message. Visualization is becoming an efficient way to convey abstract as well as concrete ideas. The key features involved in the technologies of VR and art design visualization are discussed as follows. 
2.3.1. Intuitiveness. From the primitive society to the current ear of digitization, man always strived for faster and easier availability of information. In the modern age, men are struggling for tools and techniques to more efficiently, accurately, timely, and securely operate, record, and communicate information. However, with every passing day, the quantity of data is increasing and the demand for quality is getting higher and higher. To comply with the need, the intuition of communication has been gradually strengthened. Various means have enriched the way of information transmission. Multimedia is integrated by multiple means such as text, pictures, and sound and is utilized for communication purpose. Advanced sensing tools that work on the basis of human sight, hearing. and/or touch are also used for the purpose. Moreover, comprehensive multimedia technology can be used to obtain more information resources.

Compared with previous visualization methods, modern visualization methods are mostly based on comprehensive media. It is not limited to one method nor is it limited to one medium, especially the art design that incorporates VR technology. To fully display the information of the exhibited objects and interactions, the amount of information contained in it is far greater than the traditional visualization methods.

In the virtual reality-based display design, we can combine the three-dimensional world with the design platform. With the help of sensor media, a designer may work in the virtual environment perceiving it as a real environment. People can arbitrarily specify the angle to see the visualized art image, zoom in, or zoom out arbitrarily. With the virtual reality-based display design, a designer uses the digital three-dimensional model as a display carrier. A designer may extend the artistic attribute of images in an unprecedented and intuitive way. Moreover, instead of remaining a passive observer, the designer assumes that he is inside the virtual environment.

2.3.2. Interactivity. In traditional visual design, even if the fourth dimension time is added, the model can be made merely an animation that can be demonstrated to viewers. The movement of lens is developed in accordance with the designer's thinking and not on the basis to attract viewers.

The biggest feature of VR technology is its interactivity. That is, in the VR display design, the creator abandoned the original visual route arrangement. Initiative is granted to the viewer thus making the human-computer interaction more frequent and realistic. Since people can operate as they wish in this environment, the joy of experience interaction is constantly ensured. This kind of information feedback between man and machine reflects the existence value and applicability of VR display design. The application of virtual reality technology has opened up a new technological path in information display. With the intermingling of VR in visual design, the possible barriers in image art design can be overcome and an extended and attractive expression can be achieved.
2.3.3. Authenticity. The visual design of artistic images using virtual reality technology strives to simulate an image that is as realistic as a real object. In some cases, it is treated even beyond a real thing, which is its authenticity and surreal side. If the designer can play his perceptual side in the design, it will make the display better. If the imagination is further exerted, it may also resonate with the viewers, thereby achieving the purpose of driving the viewers' emotions. The virtual digital technology has made possible the interlinking of synthetic world with the real material world. Moreover, with the realm of freedom into the world, the making of display content becomes more connotative, richer, and free from any kind of restrictions and constraints.

2.4. Dynamic Interference Detection Algorithm. The dynamic interference detection algorithm represents the artistic image as a linear combination of atoms in an incomplete dictionary composed of all training samples. The algorithm transforms the image recognition problem into a classification problem of a linear regression model. Assuming that in all $n$ training samples, the number of samples in the i-th category is $t_{i}$, and the $\omega_{i, j} \in Q^{\pi}$ vector represents the $j$-th image of the i-th category, where $m$ represents the dimension of the feature vector, and the vector set formed by all samples can be represented as follows:

$$
F_{i}=\sum W\left(\omega_{i, 1}, \omega_{i, 2}, \ldots, \omega_{i, n_{i}}\right) Q^{\pi * t_{i}} .
$$

According to the principle of linear subspace, when the sample $f$ belongs to a certain type of training samples, $k$ can be regarded as being located in the linear subspace formed by the samples of this type. Therefore, the sample $y$ can be expressed as a linear combination of the training samples of the type as follows:

$$
k=f_{i, 1} \omega_{i, 1} \oplus f_{i, 2} \omega_{i, 2} \oplus \ldots \oplus f_{i, n_{i}} \omega_{i, n_{i}} .
$$

Among them, $\omega_{i, j} \in Q$ is a scalar.

$$
E(\omega, u, i)=\frac{\sum_{\omega \in A_{u}} Q_{\omega, i}}{A+B},
$$

where $E(\omega, u, i)$ represents the target user's predicted score for item $i, A_{u}$ represents the nearest neighbor set of user $u, B$ represents the number of users in set $A_{u}$, and $Q_{\omega, j}$ represents the score of neighbor user on item $i$.

$$
S(i, j)=\frac{|B(i) \cup B(j)|}{\sqrt{B(i) \oplus B(j)}},
$$

where $S(i, j)$ represents the similarity between item $i$ and item $j, B(i)$ is the number of users who like item $i$, and $B(j)$ is the number of users who like item $i$.

\section{Experimental Details}

Normally, in visualization design, a designer plays with image. The usual artistic operation directly or indirectly performs changes in illumination, occlusion, and/or color. Aim behind the evaluation is to assess how much these 
TABLE 1: Visualization in different situations.

\begin{tabular}{|c|c|c|c|c|}
\hline Number of images & Lighting changes (\%) & Occlusion changes (\%) & Color change (\%) & No change (\%) \\
\hline 50 & 95.48 & 93.62 & 94.37 & 98.12 \\
\hline 100 & 94.49 & 93.38 & 93.48 & 97.83 \\
\hline 150 & 92.51 & 92.29 & 92.19 & 96.68 \\
\hline
\end{tabular}

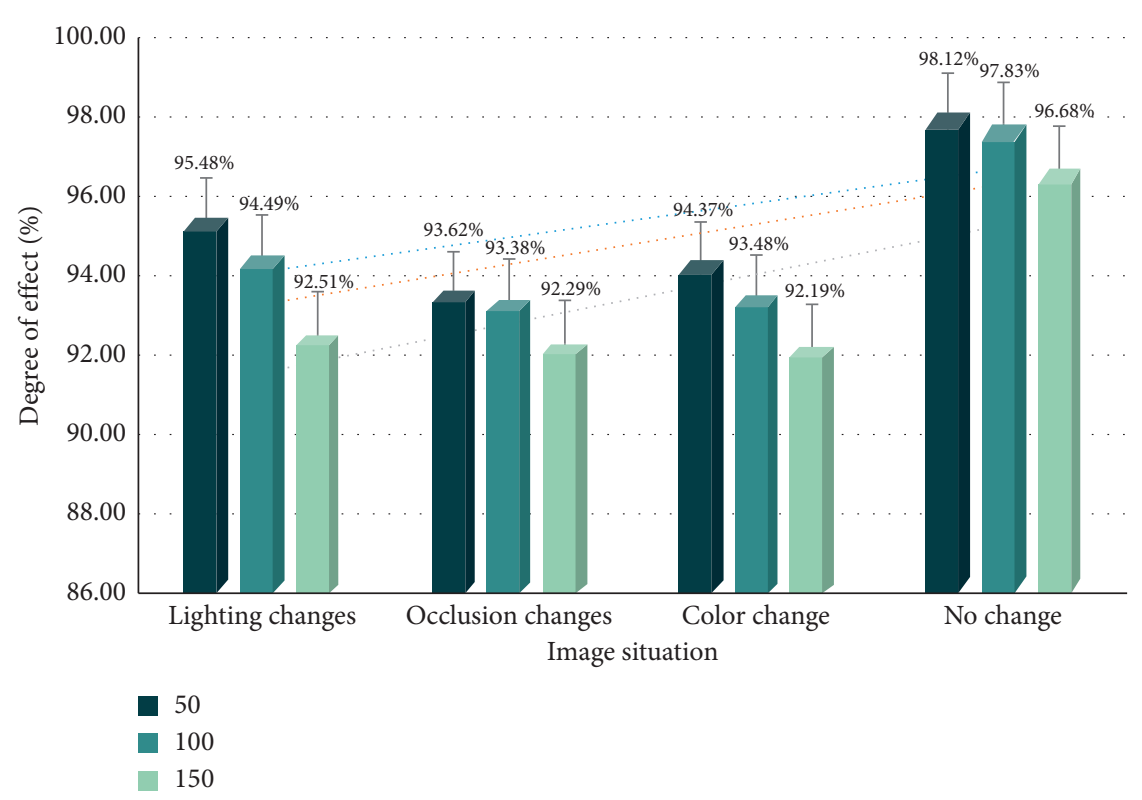

FIGURE 1: Visualization in different situations.

artistic operations are performed efficiently with the VR technology. The experimentation details are given as follows.

3.1. Experimental Data. To avoid the chances of biasness, a large sample containing 150 artistic images was obtained from an AR database. Among the images, 30 detailed images of each art image were collected in two different stages. In each of the stage, 10 images were selected including 1 frontal image, 3 light changes, 3 color changes, and 3 occluded images.

3.2. Experimental Process. Prior to the conduction of experiment as a preprocessing, the size of all the art images was normalized to $60 \times 43$ pixels. This was to ensure that same size images are to be given to both the technique for changing illumination, occlusion, and color and moreover to impartially verify that how the normal artistic design and VRbased design visualize the same size images and how the visualization is rendered. In the second phase of the experiment, comparison of the effects of visualization between the traditional image visualization and VR-based visualization is performed. By doing so, a baseline is set to further illustrate the application and advantages of VR technology in the related field.

3.2.1. Visualization in Different Situations. Three kinds of artistic image-based operations, namely, illumination change, occlusion change, and color change were performed using the ordinary artistic image design method and the VRbased approach. The visualization results obtained were compared to gauge the visual effect. VR-based visualization is up to the mark for all the three image operations as shown in Table 1 and Figure 1.

The findings exhibit that while applying the VR technology, the visualization process does not significantly affect the artistic images while performing the operations such as lighting changes, occlusion changes, and color changes. The gap is small, and the visualization effect reaches $90 \%$ or higher, showing a good visualization effect. This in turn proves that the application of VR technology in the visual design of artistic images is considerably effective.

Unlike the traditional technology, VR-based visualization is more comprehensive which is not limited to one method or one medium. Moreover, the visualization will be more effective in case of collaborative virtual environment. One of the goals of this research is to pave the way for such collaborative VR-based design where each user will be privileged to participate in the design of a realistic 3D graphics and stereo at the same time. They will be able to interact with other users through network computers besides working in a virtual environment. This will further promote the application of VR in the field of artistic design and visualization.

3.2.2. Comparison of Visual Effects of Artistic Images. In order to further illustrate the advantages of VR technology in the application of artistic image visualization design, effects 
TABLE 2: Comparison of visual effects of artistic images in two ways.

\begin{tabular}{lcccc}
\hline & Lighting changes (\%) & Occlusion changes (\%) & Color change (\%) & No change (\%) \\
\hline Virtual reality technology & 94.48 & 93.39 & 93.47 & 97.77 \\
Traditional technology & 85.57 & 88.38 & 81.13 & 89.69 \\
\hline
\end{tabular}

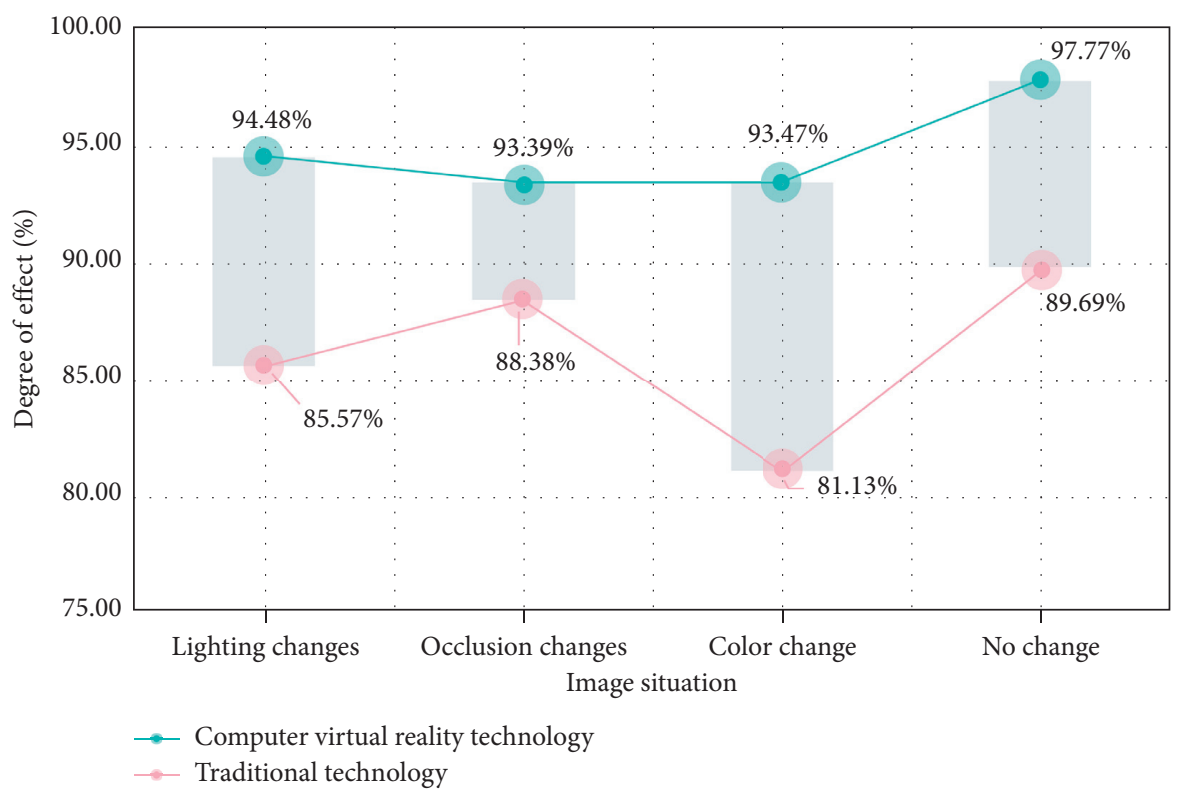

Figure 2: Comparison of visual effects of artistic images in two ways.

of the artistic image visualization products of the two approaches were compared with each other. In this experiment, images from the AR database were selected for the three image operations-illumination change, occlusion change, and color change. This time, though images were different, the number of art images was the same, i.e., 100 . Outcomes of the comparison are shown in Table 2 and Figure 2.

\section{Discussion}

Art image design is a creative discipline deals with integration of visual representation, information, and ideas. The technology of art image design is extensively in use in various disciplines like photography, calligraphy, topography, and motion graphics. Designers demand for innovation in art design to optimize and promote their work. VR is the emerging technology that realistically depicts ideas and imaginary scenario. The technology of VR is much more effective in the field of visual design of artistic images. The technology allows multiple users or multiple virtual worlds located in different physical locations to connect to a system that shares information through a network. Compared with the traditional visualization technology, it contains more information and can fully display information about the exhibitions and works. Experimental data shows that, in the face of four different artistic image situations, the effects of traditional art image visualization products produced by the art image visualization and that of VR technology are quite different. For instance, the score for the lighting changes operation using VR-based artistic design is $95.48 \%$, whereas the value $85.57 \%$ was obtained for the same operation using the traditional image design technology. For the three image-related operations, round about $10 \%$ higher scores were attained for the VR-based approach. This asserts that the VR technology can be utilized for enhancing art image design and visualization.

\section{Conclusions}

One of the key challenges in visualization and artistic image design is to find innovative way to represent graphical data in more absorbing fashion. The technology of VR comes with engrossingly representing real or imaginary scenario. This paper deals with combining the customary artistic image design with the emerging VR technology. The research work highlights application of the promising VR technology in the domain of art image visualization design. A summary is presented about the technical characteristics of modern art image visualization design and VR. The paper analyzes the possibility of combining the two technologies for effective and effortless visualization and artistic image design. Moreover, the paper reviews history and development of display design and summarizes the technical characteristics of modern art image visualization design and VR. With the help of the relevant algorithm, the association of the two areas is explored. In short, the model proposed in this paper for art image design is based on the visual interactive process, supplemented by rigorous assembly sequence and equipment mode. The whole design process is 
more controllable and feasible. After the design concept is put forward, it is verified dynamically. Through the collection and comparison of feedback information, the hypothesis algorithm is further verified and innovated. This can directly reflect the complete image design process. Finally, the study compares it with the traditional art image visualization design and proves that the VR technology is of great significance for art image visualization design. However, as the price of advanced intelligent terminal is high, challenges are there in the generalization of the model. In addition, in the art image design, typically a user selects color based on his own choice. How an intelligent device be trained to precisely express the creator's idea is a problem that needs to be addressed in order to ensure the future advancement of VR technology in the field of art image design.

\section{Data Availability}

Data sharing is not applicable to this article as no datasets were generated or analyzed during the current study.

\section{Conflicts of Interest}

The authors declare that they have no conflicts of interest.

\section{References}

[1] Z. Wang, X. Jin, and J. Pan, "Application of virtual reality technology in the career of elevator engineering\% VR technology in the teaching of elevator engineering," China Elevator, vol. 28, no. 6, pp. 62-63, 2017.

[2] G. Fan and W. Zhu, "Application of computer virtual reality technology in virtual tour," International Journal of Advanced Media and Communication, vol. 6, no. 2, pp. 273-275, 2016.

[3] P. Zheng and L. Huang, "Research on the application of 3D virtual reality technology in the protection and development of ancient villages," Revista de la Facultad de Ingenieria, vol. 32, no. 5, pp. 766-774, 2017.

[4] Le Lu, "The application of virtual reality technology in the teaching of art history," Journal of Jiamusi Education College, vol. 8, no. 12, pp. 226-227, 2017.

[5] Y. Tang, Y. Zhang, and H. Wang, "The application of virtual reality technology in con-struction engineering\% the application of virtual reality technology in con-struction engineering," Science, vol. 58, no. 6, pp. 186-188, 2018.

[6] C. J. Lin, W. J. Shiang, R. W. Wang, and T. -J. Chen, "Evaluation of virtual reality presentation in user testing procedure for product usability of a conceptual design," Computer Science, vol. 31, no. 4, pp. 307-318, 2016.

[7] W. Zhu and G. Fan, "Application of computer virtual reality technology in virtual tour," International Journal of Advanced media and Communication, vol. 6, no. 2, pp. 273-282, 2016.

[8] C. Zhang, "Research on interface visual communication design based on virtual reality technology in paper manufacturing," Paper Asia, vol. 2, no. 1, pp. 146-150, 2019.

[9] A. K. Yetisen, A. F. Coskun, G. England et al., "Art on the nanoscale and beyond," Advanced Materials, vol. 28, no. 9, pp. 1724-1742, 2016.

[10] J. Yang, "Design of coal mine three-dimensional visual display system based on virtual reality technology," Shaanxi Coal, vol. 38, no. 4, pp. 127-129, 2019.
[11] L. Dong, "Application of computer virtual reality technology in civil engineering," Computer products and circulation, vol. 5, no. 4, pp. 25-28, 2020.

[12] D. Han, "Analysis of the application of virtual reality technology in environmental art design," Building development, vol. 4, no. 4, pp. 8-12, 2020.

[13] S. Huang, "Application Research of virtual reality technology," Decision making exploration, vol. 11, no. 2, pp. 14-16, 2020. 